\title{
Using Dialogue Representations for Concept-to-Speech Generation
}

\author{
Christine H. Nakatani \\ Jennifer Chu-Carroll \\ Bell Laboratories, Lucent Technologies \\ 600 Mountain Avenue \\ Murray IIill, NJ 07974 USA \\ $\{$ chnljencc\}oresearch.bell-labs.com
}

\begin{abstract}
We present an implemented concept-to-speech (CTS) system that offers original proposals for certain couplings of dialogue computation with prosodic computation. Specifically, the semantic interpretation, task modeling and dialogue strategy modules in a working spoken dialogue system are used to generate prosodic features to better convey the meaning of system replies. The new CTS system embodies and extends theoretical work on intonational meaning in a more general, robust and rigorous way than earlier approaches, by reflecting compositional aspects of both dialogue and intonation interepretation in an original computational framework for prosodic generation.
\end{abstract}

\section{Introduction}

Conversational systems that use speech as the input and output modality are often realized by architectures that decouple speech processing components from language processing components. In this paper, we show how speech generation can be more closely coupled with the dialogue manager of a working mixed-initiative spoken dialogue system. In particular, we use representations from the semantic interpretation, task model and dialogue strategy modules to better communicate the meaning of system replies through prosodically appropriate synthetic speech.

While dialogue prosody has been a topic of much study, our implemented concept-to-speech (CTS) system offers original proposals for specific couplings of dialogue computation with prosodic computation. Further, it embodies and extends theoretical work on intonational meaning in a more general, robust and rigorous way than earlier CTS systems, in an architecture that reflects compositional aspects of dialogue and intonation interpretation.

\section{Theoretical Foundations}

In this work, we implement and extend the compositional theory of intonational meaning proposed by Pierrehumbert and Hirschberg (1986; 1990), who sought to identify correspondences between the
Grosz and Sidner (1986) computational model of discourse interpretation and Pierrehumbert's prosodic grammar for American English (1980).

In the present work, certain aspects of the original theories are modified and adapted to the architecture of the dialogue system in which the CTS component is embedded. Below, we present the important fundamental definitions and principles of intonation underlying our CTS system.

\subsection{Intonational System}

In our CTS system, the prosodic elements that are computed are based on the intonational system of Pierrehumbert (1980), who defined a formal language for describing $\Lambda$ merican English intonation using the following regular grammar:

Inton Phrase $\rightarrow$ (Interm Phrase) $^{+}$Bndry Tone Interm Phrase $\rightarrow(\text { Pitch Acc })^{+}$Phrase Acc

Major phrases, or intonational phrases, are made up of one or more minor phrases, or intermediate phrases. Melodic movements in intermediate and intonational phrases are in turn expressed by three kinds of tonal elements. These include six pitch accents: a low pitch excursion $\left(L^{*}\right)$, a high pitch excursion $\left(\mathrm{H}^{*}\right)$, or a combination of both low and high excursions $\left(\mathrm{L}^{*}+\mathrm{H}, \mathrm{L}+\mathrm{H}^{*}, \mathrm{H}^{*}+\mathrm{L}, \mathrm{H}+\mathrm{L}^{*}\right)$; two phrase accents: a high ( $\mathrm{H}-$ ) or low ( $\left.\mathrm{L}^{-}\right)$tonal target that guides the interpolation of the melodic contour from final pitch accent to intermediate phrase ending; and two boundary tones: a high (H\%) or low (L\%) tonal target that guides interpolation from phrase accent to intonational phrase ending.

\subsection{Intonational Meaning}

Theoretical work on intonational meaning has attempted to relate the grammatical elements of Pierrehumbert's system - pitch accent, phrase accent and boundary tone, to interpretive processes at different levels of discourse and dialogue structure. Hirschberg and Pierrehumbert (1986) conjectured that the absence or presence of accentuation conveys discourse focus status, while the tonal properties of the accent itself (i.e. pitch accent type) convey semantic focus information. 
MIMIC: hello this is mimic the movie information system

how can I help you

User: where in hoboken is october sky playing

MIMIC: october sky is playing at hoboken cinema in hoboken

can I help you with anything else

User: when is it playing there

MIMIC: october sky is playing at hoboken cinema in hoboken at $3: 45 \mathrm{pm}, 5: 50 \mathrm{pm}, 7: 10 \mathrm{pm}$, and $10 \mathrm{pm}$

can i help you with anything else

Figure 1: A MIMIC dialogue.

In later work, pitch accent type was said to express whether the accented information was intended by the speaker to be "predicated" or not by the hearer (Pierrehumbert, and Hirschberg, 1990). Non-predicated-information was said to bear lowstar accentuation $\left(\mathrm{L}^{*}, \mathrm{~L}^{*}+\mathrm{Il}, \mathrm{H}+\mathrm{L}^{*}\right)$, while predicated information would be marked by high-star accents $\left(\mathrm{H}^{*}, \mathrm{~L}+\mathrm{H}^{*}, \mathrm{H}^{*}+\mathrm{L}\right)$. The theory further stated that $\mathrm{L}^{*}+\mathrm{H}$ conveys uncertainty or lack of speaker commitment to the expressed propositional content, while $\mathrm{L}+\mathrm{H}^{*}$ marks correction or contrast. The complex accent, $\mathrm{H}^{*}+\mathrm{L}$, was said to convey that an inference path was required to support the predication; usage of $\mathrm{H}+\mathrm{L}^{*}$ similarly was said to imply an inference path, but did not suggest a predication of a mutual belief. Finally, phrase accents and boundary tones were said to reflect aspects of discourse structure.

\section{Systems Foundations}

Our task is to improve the communicative competence of a spoken dialogue agent, by making recourse to our knowledge of intonational meaning, dialogue processing and relations between the two. Of course, a worthwhile CTS system must also outperform out-of-the-box text-to-speech (TTS) systems that may determine prosodic mark-up in linguistically sophisticated ways. As in (Nakatani, 1998), we take the prosodic output of an advanced research system that implements the Pierrehumbert theory of intonation, namely the Bell Labs TTS system, as our baseline experimental system to be enhanced by CTS algorithms. We embed the CTS system in MIMIC, a working spoken dialogue system representing state-of-the-art dialogue management practices, to develop CTS algorithms that can be eventually realistically evaluated using task-based performance metrics.

\subsection{Dialogue System: Mixed-Initiative Movie Information Consultant (MIMIC)}

The dialogue system whose baseline speech generation capabilities we enhance is the MixedInitiative Movie Information Consultant (MIMIC) (Chu-Carroll, 2000). MIMIC provides movie list- ing information involving knowledge about towns, theaters, movies and showtimes, as demonstrated in Figure 1. MIMIC currently utilizes templatedriven text generation, and passes on text strings to a stand-alone TTS system. In the version of MIMIC enhanced with concept-to-speech capabilities, MIMIC-CTS, contextual knowledge is used to modify the prosodic features of the slot and filler material in the templates; we are currently integrating the algorithms in MIMIC-CTS with a grammardriven generation system. Further details of MIMIC are presented in the relevant sections below, but see (Chu-Carroll, 2000) for a complete overview.

\subsection{TTS: The Bell Labs System}

For default prosodic processing and speech synthesis realization, we use a research version of the Bell Labs TTS System, circa 1992 (Sproat, 1997), that generates intonational contours based on Pierrehumbert's intonation theory (1980), as described in (Pierrehumbert, 1981). Of relevance is the fact that various pitch accent types, phrase accent and boundary tones in Pierrehumbert's theory are directly implemented in this system, so that by generating a Pierrehumbert-style prosodic transcription, the work of the CTS system is done. More precisely, MIMIC-CTS computes prosodic annotations that override the default prosodic processing that is performed by the Bell Labs TTS system.

To our knowledge, the intonation component of the Bell Labs TTS system utilizes more linguistic knowledge to compute prosodic annotations than any other unrestricted TTS system, so it is reasonable to assume that improvements upon it are meaningful in practice as well as in theory.

\section{MIMIC's Concept-to-Speech Component (MIMIC-CTS)}

In MIMIC-CTS, the MIMIC dialogue system is enhanced with a CTS component to better communicate the meaning of system replies through contextually conditioned prosodic features. MIMIC-CTS makes use of three distinct levels of dialogue representations to convey meaning through intonation. MIMIC's semantic representations allow MIMICCTS to decide which information to prosodically 
highlight. MIMIC's task model in turn determines how to prosodically highlight selected information, based on the pragmatic properties of the system reply. MIMIC's dialogue strategy selection process informs various choices in prosodic contour and accenting that convey logico-semantic aspects of meaning, such as contradiction.

\subsection{Highlighting Information using Semantic Representations}

MIMIC employs a statistically-driven semantic interpretation engine to "spot" values for key attributes that make up a valid MIMIC query in a robust fashion. ${ }^{1}$ To simplify matters, for each utterance, MIMIC computes an attribute-value matrix (AVM)-representation, identifying important pieces of information for accomplishing a given set of tasks. The AVM created from the following utterance, "When is October Sky playing at Hoboken Cinema in Hoboken?", for example, is given in Figure 2 .

\begin{tabular}{|l||l|}
\hline Attribute & Value \\
\hline \hline Task & when \\
Movie & October Sky \\
Theatre & Hoboken Cinema \\
Town & Hoboken \\
Time & - \\
\hline
\end{tabular}

Figure 2: Attribute Value Matrix (AVM), computed by MIMIC's semantic interpreter.

Attribute names and attribute values are critical to the task at hand. In MIMIC-CTS, attribute names and values that occur in templates are typed, so that MIMIC-CTS can highlight these items in the following way:

1. All lexical items realizing attribute values are accented.

2. Attribute values are synthesized at a slower speaking rate.

3. Attribute values are set off by phrase boundaries.

4. Attribute names are always accented.

These modifications are entirely rule-based, given a list of attribute names and typed attribute values.

\footnotetext{
${ }^{1}$ Specifically, MIMIC uses an $n$-dimensional call router front-end (Chu-Carroll, 2000), which is a generalization of the vector-based call-routing paradigm of semantic interpretation (Chu-Carroll and Carpenter, 1999); that is, instead of detecting one concept per utterance, MIMIC's semantic interpretation engine detects multiple $(n)$ concepts or classes conveyed by a single utterance, by using $n$ call routers in parallel.
}

Even such minimal use of dialogue information can make a difference. For example, changing the default accent for the following utterance highlights the kind of information that the system is seeking, instead of highlighting the semantically vacuous main verb, like: ${ }^{2}$

\section{Default TTS: what movie would you LIKE} MIMIC-CTS: what MOVIE would you like

\subsection{Conveying Information Status using the Task Model}

MIMIC performs a set of information-giving tasks, i.e. what, where, when, location, that are concisely defined by a task model. MIMIC processes the AVM for each utterance and then evaluates whether it should perform a database query based on the task specifications given in Figure 3 . The task model defines which attribute values must be filled in (Y), must not bë filled in $(\mathrm{N})$, or may optionally be filled in (-), to "license" a database query action. If no task is "specified" by the current AVM state,

\begin{tabular}{|l||l|l|l|}
\hline Task & Movie & Theater & Town \\
\hline \hline What & $\mathrm{Y}$ & $\mathrm{Y}$ & - \\
Where & $\mathrm{Y}$ & $\mathrm{N}$ & $\mathrm{Y}$ \\
When & $\mathrm{Y}$ & $\mathrm{Y}$ & - \\
\hline
\end{tabular}

Figure 3: Task Specifications for MIMIC.

MIMIC employs various strategies to progress toward a complete and valid task specification. For example, in response to the following user utterance, MIMIC initiates an information-seeking subdialogue to instantiate the theater attribute value to accomplish a when task:

User: when is october sky playing in hoboken

MIMIC-CTS: what THEATER would you like

To better convey the structure of the task model, which is learned by the user through interaction with the system, we define four information statuses based on properties of the task model, which align on a scale of given and new in the following order:

$$
\begin{array}{lrr}
\text { OLD } & \text { INFERRABLE } & \text { KEY } \\
\text { [given] } & \text { HEARER-NEW } \\
\text { [new] }
\end{array}
$$

KEY information is that which is necessary to formulate a valid database query, and is exchanged and (implicitly or explicitly) confirmed between the system and user. INFERRABLE information is not explicitly exchanged between the system and

\footnotetext{
${ }^{2}$ In the examples, small capitalization denotes a word is accented.
} 


\begin{tabular}{|l|l|l|}
\hline Task Specification Status & Information Status & Pitch Accent \\
\hline Required (Y) & KEY & $\mathrm{L}+\mathrm{H}^{*}$ \\
Optional $(-)$ & INFERRABLE/OLD & $\mathrm{L}^{*}+\mathrm{H} / \mathrm{L}^{*}$ \\
Not allowed $(\mathrm{N})$ & HEARER-NEW & $\mathrm{H}^{*}$ \\
\hline
\end{tabular}

Table 1: Highlighting relevance of information based on task model (and discourse history).

User: where in montclair is analyze this playing

MIMIC: analyze this is playing at wellmont theatre and clearviews screening zone in montclair

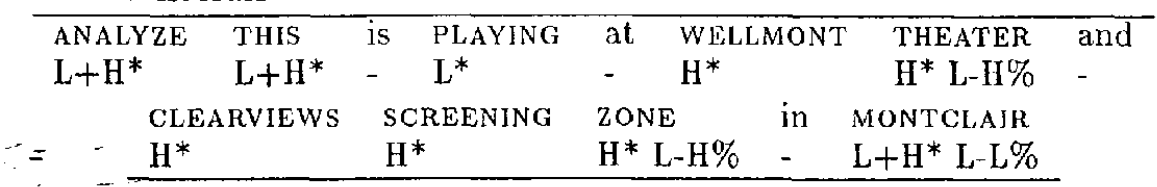

Figure 4: Above, dialogue excerpt of MIMIC performing a where task. Below, the modified version of the bold-faced reply string, generated by MIMIC-CTS.

user, but is derived by MIMIC's limited inference engine that seeks to instantiate as many attributc values as possible. For instance, a theater name may be inferred given a town name, if there is only one theater in the given town. OLD information is inherited from the discourse history, based on updating rules relying on confidence scores for attribute values. HEARER-NEW information (c.f. (Prince, 1988)) is that which is requested by the user, and constitutes the only new information on the scale. But note that KEY information, while given, is still clearly in discourse focus, along with HEARER-NEW information.

The next step is to map the information statuses, ordered from given to new, to a scale of pitch accent, or accent melodies, ordered from given to new as follows:

$$
\begin{array}{llrr}
\mathrm{L}^{*} & \mathrm{~L}^{*}+\mathrm{H} & \mathrm{L}+\mathrm{H}^{*} & \mathrm{H}^{*} \\
\text { [given] } & & & {[\text { new] }}
\end{array}
$$

Table 1 summarizes this original mapping of information statuses to pitch accent melodies, and Figure 4 illustrates the use of this mapping in an example. It obeys the general principle of Pierrehumbert and Hirschberg's work, that low tonality signifies discourse givenness and high tonality signifies discourse newness, but extends this principle beyond its vague definition in terms of predication of mutual beliefs. Instead, the principle is operationalized here in a practically motivated manner that is consistent with and perhaps illuminating of the theory.

\subsection{Assigning "Dialogue Prosody" using Dialogue Strategies}

$\Lambda \mathrm{s}$ in earlier CTS systems, special logico-semantic relations, such as contrast or correction, are effectively conveyed in MIMIC-CTS by prosodic cues. In MIMIC-CTS, however, these situations are not stipulated in an ad hoc manner, but can be determined to a large degree by MIMIC's dialogue strategy selection process that identifies appropriate dialogue acts to realize a dialogue goal. ${ }^{3}$

For example, the dialogue act ANSWER may be selected to achieve the dialogue goal of providing an answcr to a successful user query, while the dialogue act Notify FalluRe may be performed to achieve the dialogue goal of providing an answer in situations where no movie listing in the database matches the user query. The template associated with the dialogue act, Notify FaILURE, when compared with that for ANSWER, contains an additional negative auxiliary associated with the key attribute responsible for the query failure, in an utterance conveying a contradiction in beliefs between the user and system (namely, the presupposition on the part of the user that the query can be satisfied).

Theoretical work on intonational interpretation leads us to prosodically mark the negative auxiliary, as well as the associated focus position (Rooth, 1985). We choose to mark the negative auxiliary not with the $\mathrm{L}+\mathrm{H}^{*}$ pitch accent to convey correction, while marking the material in the associated focus position with the $\mathrm{L}^{*}+\mathrm{H}$ pitch accent to convey (the

\footnotetext{
${ }^{3}$ Importantly, MIMIC's adaptive dialogue strategy selection algorithm takes into account the outcome of an initiative tracking module that we do not discuss here (see (ChuCarroll, 2000)).
} 


\begin{tabular}{|c|c|c|c|c|c|c|}
\hline $\begin{array}{l}\text { User: } \\
\text { MIMIC }\end{array}$ & $\begin{array}{l}\text { where is tl } \\
\text { the corru } \\
\text { the corrup }\end{array}$ & or & $\begin{array}{l}\text { ruptor } \\
\text { is not } \\
\text { playing }\end{array}$ & $\begin{array}{l}\text { laying in } \\
\text { laying } \mathrm{i} \\
\text { at lincoln }\end{array}$ & & $\begin{array}{l}\text { ord } \\
\text { inford } \\
\text { mas in arlington }\end{array}$ \\
\hline $\begin{array}{l}\mathrm{THE} \\
\mathrm{L}+\mathrm{H}^{*}\end{array}$ & $\begin{array}{l}\text { CORRUPTOR } \\
\mathrm{L}+\mathrm{H}^{*}\end{array}$ & is & $\begin{array}{l}\text { NOT } \\
\mathrm{L}+\mathrm{H}^{*}\end{array}$ & $\begin{array}{l}\text { playing } \\
! \mathrm{H}^{*}\end{array}$ & in & $\begin{array}{l}\text { CRANFORD } \\
\mathrm{L}^{*}+\mathrm{H} \text { L-H } \%\end{array}$ \\
\hline
\end{tabular}

Figure 5: Above, dialogue excerpt of MIMIC performing a Notify FaILURE dialogue act. Below, the modified version of the bold-faced reply string, generated by MIMIC-CTS. Note the diacritic "!" denotes a downstepped accent (see (Pierrehumbert, 1980)).

system's) lack of commitment to the (user's) presupposition at hand. Finally, the Notify Fallure dialogue act is conveyed by assigning the so-called rise-fall-rise contradiction contour, $\mathrm{L}^{*}+\mathrm{H} \mathrm{L}-\mathrm{H} \%$, to the utterance at large (c.f. (Hirschberg and Ward, 1991)). An example generated by MIMIC-CTS appears in Figure 5. Note that pitch accent types for the remaining attribute values are assigned using the task model, as described in section 4.2. Thus in Figure 5 , the movie title is treated as KEY information, marked by the $\mathrm{L}+\mathrm{H}^{*}$ pitch accent.

MIMIC-CTS contains additional prosodic rules for logical connectives, and clarification and confirmation subdialogues.

\section{Related Work}

Although a number of earlier CTS systems have captured linguistic phenomena that we address in our work, the computation of prosody from dialogue representations is often not as rigorous, detailed or complete as in MIMIC-CTS. Further, while several systems use given/new information status to decide whether to accent or deaccent a lexical item, no system has directly implemented general rules for pitch accent type assignment. Together, MIMIC-CTS's computation of accentuation, pitch accent type and dialogue prosody constitutes the most general and complete implementation of a compositional theory of intonational meaning in a CTS system to date.

Nevertheless, elements of a handful of previous CTS systems support the approaches taken in MIMIC-CTS toward conveying semantic, task and dialogue level meaning. For example, the Direction Assistant system (Davis and Hirschberg, 1988) mapped a hand-crafted route grammar to a discourse structure for generated directions. The discourse structure determined accentuation, with deaccenting of discourse-old entities realized (by lexically identical morphs) in the current or previous discourse segment. Other material was assigned accentuation based on lexical category information, with the exception that certain contrastive cases of accenting, such as left versus right, were stipulated for the domain.

Accent assignment in the SUNDIAL travel infor- mation system (House and Youd, 1990) also relied on discourse and task models. Mutually known entities, said to be in negative focus, were deaccented; entities in the current task space, in referring focus, received (possibly contrastive) accenting; and entities of the same type as a previously mentioned object, were classified-as in either referring or emphatic focus, depending on the dialogue act: In the cases of corrective situations or repeated system-intitiated queries, the contrasting or corrective items were emphatically accented.

The BRIDGE project on speech generation (Zacharski et al., 1992) identified four main factors affecting accentability: linear order, lexical category, semantic weight and givenness. In related work (Monaghan, 1994), word accentability was quantitatively scored by hand-crafted rules based on information status, semantic focus and word class. The givenness hierarchy of Gundel and colleagues (1989), which associates lexical forms of expression with information statuses, was divided into four intervals, with scores assigned to each. A binary semantic focus score was based on whether the word occurred in the topic or comment of a sentence. Finally, lexical categories determined word class scores. These scores were combined, and metrical phonological rules then referred to final accentability scores to assign $a$ final accenting pattern.

To summarize, all of the above CTS systems employ either hand-crafted or heuristic techniques for representing semantic and discourse focus information. Further, only SUNDIAL makes use of dialogue acts.

\section{Conclusion and Future Work}

We are presently carrying out evaluations of MIMICCTS. An initial corpus-based analysis compares the prosodic annotations assigned to three actual MIMIC dialogues, which were previously collected during an overall system evaluation (ChuCarroll and Nickerson, 2000). The corpus of dialogues is made up of 37 system/user turns, including 40 system-generated sentences. Three versions of the MIMIC dialogues are being analysed, with prosodic features arising from three differ- 
ent sources: MIMIC-CTS, MIMIC operating with default Bell Labs TTS, and a professional voice talent who read the dialogue scripts in context. This corpus-based assessment --- comparing the prosody of CTS-generated, TTS-generated, and human speech, will enable more domain-dependent tuning of the MIMIC-C'IS algorithms, as well as the refinement of general prosodic patterns for linguistic structures, such as lists and conjunctive phrases. Ultimately, the value of MIMIC-CTS must be measured based on its contribution to overall task peformance by real MIMIC users. Such a study is under design, following (Chu-Carroll and Nickerson, 2000).

In conclusion, we have shown how prosodic computation can be conditioned on various dialogue represcntations, for robust and domain-independent CTS synthesis. - While some rules for prosody assignment depend on the task model, others must be tied closely to the particular choices of content in the replies, at the level of dialogue goals and dialogue acts. At this level as well, however, linguistic principles of intonation interpretation can be applied to determine the mappings. In sum, the lesson learned is that a unitary notion of "concept" from which we generate a unitary prosodic structure, does not apply to state-of-the-art spoken dialogue generation. Instead, the representation of dialogue meaning in experimental architectures, such as MIMIC's, is compositional to some degree, and we take advantage of this fact to implement a compositional theory of intonational meaning in a new concept-to-speech system, MIMIC-C'TS.

\section{References}

Jennifer Chu-Carroll and Bob Carpenter. 1999. Vector-based natural language call routing. Computational Linguistics, 25(3):361-388.

Jennifer Chu-Carroll and Jill S. Nickerson. 2000. Evaluating automatic dialogue strategy adaptation for a spoken dialogue system. In Proceedings of the 1st Conference of the North American Chapter of the Association for Computational Linguistics, Seattle.

Jennifer Chu-Carroll. 2000. Mimic: an adaptive mixed initiative spoken dialogue system for information queries. In Proceedings of the 6th Conference on Applied Natural Language Processing, Seattle.

J. R. Davis and J. Hirschberg. 1988. Assigning intonational features in synthesized spoken directions. In Proceedings of the 26th Annual Meeting of the Association for Computational Linguistics, pages 187-193, Buffalo.

Barbara Grosz and Candace Sidner. 1986. Attention, intentions, and the structure of discourse. Computational Linguistics, 12(3):175-204.
J. Gundel, N. Hedberg, and R."Zacharski. 1989. Givenness, implicature and demonstrative expressions in English discourse. In Proceedings of CLS25, Parasession on Language in Context, pages 89-103. Chicago Linguistics Society.

Julia Hirschberg and Janet Pierrehumbert. 1986. The intonational structuring of discourse. In Proceedings of the 24th Annual Meeting of the Association for Computational Linguistics, New York.

J. Hirschberg and G. Ward. 1991. The influence of pitch range, duration, amplitude, and spectral features on the interpretation of $\mathbf{l}^{*}+\mathbf{h} \mathbf{l} \mathbf{h} \%$. Journal of Phonetics.

Jill House and Nick Youd. 1990. Contextually appropriate intonation in speech synthesis. In Proceedings of the Furopean Spech Communication Association Workshop on Speech Synthesis, pages 185-188, Autrans.

A. I. C. Monaghan. 1994. Intonation accent placement in a concept-to-dialogue system. In Proceedings of the ESCA/IEEE Workshop on Speech Synthesis, pages 171-174, New Paltz, NY.

C. H. Nakatani. 1998. Constituent-based accent prediction. In Proceedings of the 36th Annual Meeting of the Association for Computational Linguistics, Montreal.

J. Pierrehumbert and J. Hirschberg. 1990. The meaning of intonational contours in the interpretation of discourse. In Intentions in Communication. MIT Press, Cambridge, MA.

Janet Pierrehumbert. 1980. The Phonology and Phonetics of English Intonation. Ph.D. thesis, Massachusetts Institute of Technology, September. Distributed by the Indiana University Linguistics Club.

J. Pierrehumbert. 1981. Synthesising intonation. Journal of the Acoustical Society of America, 70(4):985-995.

Ellen Prince. 1988. The ZPG letter: subjects, definiteness, and information status. In S. Thompson and W. Mann, editors, Discourse Description: Diverse Analyses of a Fund Raising Text. Elsevier Science Publishers, Amsterdam.

Mats Rooth. 1985. Association with Focus. Ph.D. thesis, University of Massachusetts, Amherst MA.

Richard Sproat, editor. 1997. Multilingual Textto-Speech Synthesis: The Bell Labs Approach. Kluwer Academic, Boston.

Ron Zacharski, A. I. C. Monaghan, D. R. Ladd, and Judy Delin. 1992. BrIDge: Basic research on intonation for dialogue generation. Technical report, University of Edinburgh. 